\title{
High Color-Rendering White Organic Light-Emitting Diodes for the Application of Lightening
}

\author{
Chun-Ya $\mathrm{LI}^{1, \mathrm{a}}$, Jie $\mathrm{LIU}^{2}$, Ming $\mathrm{LI}^{1}$, Kun-Ping $\mathrm{GUO}^{3}$, Bin WEI${ }^{3}$, and Xing-Wei \\ $\mathrm{DING}^{1, *}$ \\ ${ }^{1}$ Key Laboratory of Advanced Display and System Applications, Ministry of Education, Shanghai \\ University, Shanghai 200072, China \\ ${ }^{2}$ Langfang Polytechnic Institute, Langfang 065001, Hebei, China; \\ ${ }^{3}$ School of Mechatronic Engineering and Automation, Shanghai University, Shanghai, 200072, \\ China \\ alichunya@shu.edu.cn, *dingxingwei@163.com
}

Keywords: White OLEDs, Color rendering index, Lightening.

\begin{abstract}
The development of white organic light emitting diodes (WOLEDs) holds great promise since they combine the potentialities of high efficiency and inexpensive production with the appealing features of high-color-quality white light. Nevertheless, reliability and color quality still need to be optimized to make WOLEDs commercially competitive as alternative lighting sources. Development of stable and high color-rendering index emitters plays a key role in the progress of WOLED manufacturing. In the paper, we have investigated several approaches to achieving high color-rendering WOLEDs for solid-state lighting. A light-emitting layer (EML) of WOLED, consisting of $8 \mathrm{wt} \%$ Pt-4-doped 1,3-bis(9-carbazolyl) benzene (mCP), was very stable and shifted slightly from $(0.312,0.398)$ at $1000 \mathrm{~cd} \mathrm{~m}^{-2}$ to $(0.320,0.395)$ at $9000 \mathrm{~cd} \mathrm{~m}^{-2}$.
\end{abstract}

\section{Introduction}

The concept of organic electroluminescent (EL) can be traced back to its history since 1987, when Dr. C. W. Tang fabricated the first efficient organic devices from aluminum tris(8-quinolinolato) (Alq3) [1]. Since then, more and more organic compounds were adopted for lighting applications [2, 3]. One device on the cusp of widespread use is the organic light-emitting diodes (OLEDs) as they are low-cost, easily processable, lightweight and compatible with flexible substrates $[4,5]$. Research over the last decade has paved the way for the implementation of efficient red, green, and blue OLEDs in passive and active matrix displays [6-8]. At the meanwhile, many efforts have been paid to the influencing mechanism of the light emission property, since the band structure could be modulated by interface strain or phase transition[9-10]. Interest in the application of white organic light-emitting diode (WOLED) technology are of great importance, not only because WOLED are highly efficient and have longer lifetime than incandescent bulbs, but particularly because they have the possession of high-quality white-light illumination [11-13].

There are two critical parameters that define the color quality of a white light source: the Commision Internationale de l'Éclairage (CIE) chromaticity coordinates [14], and the color-rendering index (CRI) [15]. In order for light to be perceived as white by the human eye, two important CIE standard illuminants are "colorless" white light [corresponding to CIE coordinates of $(0.33,0.33)]$ and warm light [corresponding to CIE coordinates of $(0.448,0.408)]$, respectively [16]. Equally important as its chromaticity is the ability of a light source to reproduce the color of objects. The so-called CRI, which is a dimensionless value ranging from 0 to 100 , usually serves as a measure for this property. A CRI $\geq 80$ is required for indoor-lighting applications [16, 17]. To meet these requirements, a lot of efforts have been devoted to fabricate WOLED. For example, Kido et al. reported white light-emitting organic electroluminescent devices using the poly (N-vinylcarbazole) emitter layer doped with three fluorescent dyes. White emission covers a wide range of the visible region [18]. Wang et al. reported a WOLED with CIE coordinates of $(0.38,0.43)$ by co-doping blue 
and orange dyes into a single host material [19]. Jabbour et al. reported an excimer-based WOLED with a CRI of 81 and CIE coordinates of $(0.40,0.43)$ at $500 \mathrm{~cd} \mathrm{~m}^{-2}$ by doping a platinum (II) complex into a novel host material [20].

In the following sections we review some current developments in the exciting field of WOLEDs, with a special focus on approaches to the emission color and high CRI WOLED manufacturing. This review begins by discussing different color-conversion approaches to generate white light with organic semiconductors (Section 2). We then discuss recent trends in the development of excimer-based WOLEDs (Section 3). Different device configurations, in particular three primary colors (R, G, and B) based techniques, are introduced and some new concepts developed will be briefly reviewed (Section 4). The review closes with a brief overview of the high CRI of multiple peak emissions for WOLEDs.

\section{Advanced Color Conversion Method}

As a source of white light, an ideal WOLED should emit a continuous spectrum covering the entire visible range (400-800 nm) and should suppress the color shift. To date, extensive studies have been carried out on the fabrication of WOLEDs by various approaches such as a single emissive layer structure doped with different fluorescent material [21, 22], stacked multi-emissive layers structure in which each layer emits different colors to generate white emission [23, 24], a stacked structure WOLED [25] and a tandem cell structure [26]. To avoid chromatic instability from the emissive layers, a simple method for fabricating WOLEDs has been developed by using color conversion materials (CCMs) on the inverse glass side to a blue-emitting device [27]. Unfortunately, this approach sets limits in high-efficiency and high-purity WOLEDs, particularly for the application in full-color flat-panel displays. Very recently, a simple solution process has been used for the fabrication of a chromatic-stable WOLED, in which a red fluorescent polymer poly(2-methoxy-5-[2'-ethylhexyloxy]-p- phenylenevinylene (MEH-PPV) was developed to function as both a color conversion layer (CCL) and a hole injection layer (HIL) into the device [28].
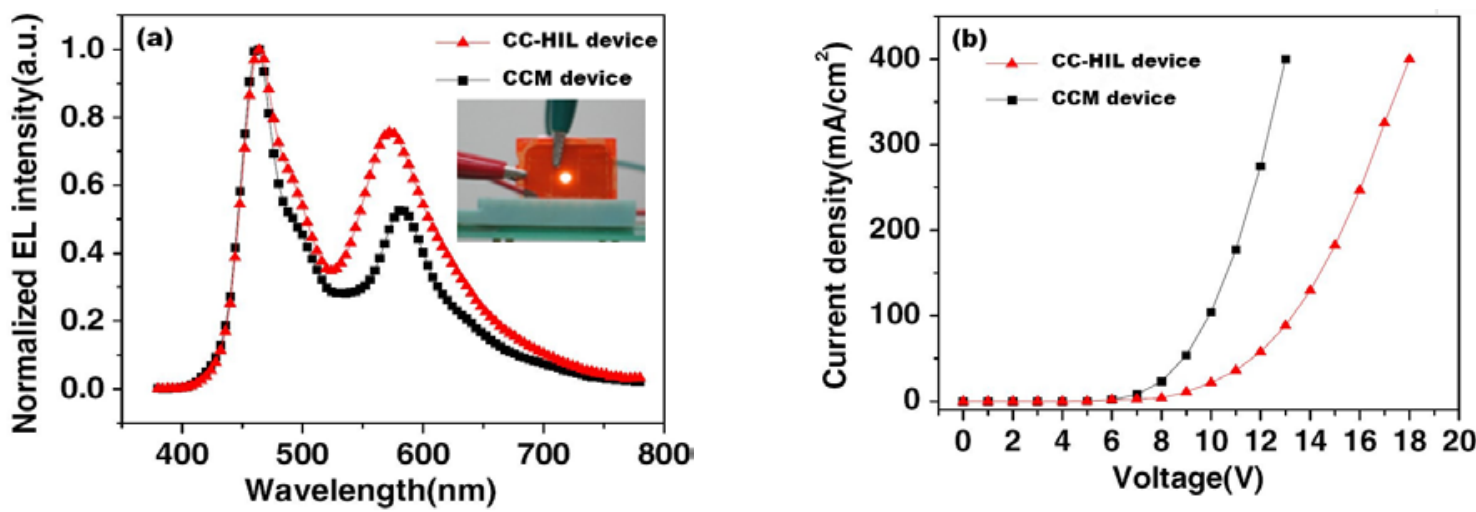

Fig. 1. (a) EL spectra of down-conversion CC-HIL WOLED and CCM-WOLED. (b) Current density-voltage characteristics of down-conversion CC-HIL WOLED and CCM-WOLED.

The EL spectra of CC-HIL device and CCM device at a current density of $100 \mathrm{~mA} \mathrm{~cm}$ are illustrated in figure 1 , corresponding to CIE coordinates of $(0.329,0.347)$ and $(0.297,0.304)$, respectively. The total output emission spectrum is a mixture of the blue EL and red PL. Compared with CCM device, the red EL intensity of CC-HIL intensity is slightly higher. It is apparent that the CC-HIL device exhibits a better performance, which should be ascribed to the smoother hole injection and enhanced light absorption efficiency. The color conversion HIL can effectively absorb the ITO/organic-guided modes and convert into low-energy PL emission.

Regardless of the specific scheme, CC-HIL approach is generally considered to offer a higher chromatic-stable and simpler device fabrication than other approaches described below. Nevertheless, fabricating organic layer from solution is easily dissolved when the subsequent layer is deposited. As 
discussed below, if all components required to generate white light are mixed into one material, one can fabricate a WOLED with a single active layer that is sandwiched between electrodes. Thus reduces the number of processing steps for device and allows straightforward deposition of the active layer using conventional vacuum thermal evaporation processes.

\section{Excimer-Based Single Molecular}

The major disadvantage associated with the single-layer concept is that one has to compromise on certain material characteristics in order to be able to combine different functionalities in a single material [15]. One promising approach to reducing the number of dopants and structural heterogeneities inherent in the multiple layer architecture is to employ a lumophore that forms a broadly emitting exciplex [10]. The excimer is only bound in the excited state and has no ground absorption, hence providing a unique solution to achieving efficient energy transfer from the host to the light-emitting centers. [29]. For example, platinum [1,3-difluoro-4,6-di(2-pyridinyl) benzene] chloride (Pt-4), produces light blue-monomer and red-excimer emission in both PL and EL. However, square-planar Pt complexes are known to form excimers when positioned in close proximity in concentrated solutions and thin films [30-32].

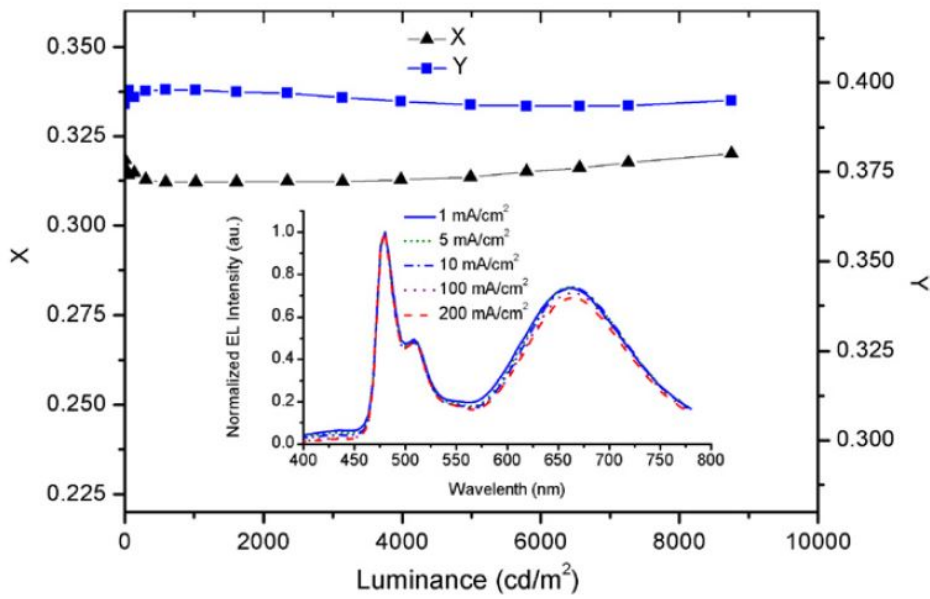

Fig. 2. Changes in CIE coordinates (X, Y) of the WOLED with luminance. The inset is the normalized EL spectra of the WOLED at different current densities.

A key feature of the device in Fig. 2 is the light-emitting layer (EML), consisting of $8 \mathrm{wt} \%$ Pt-4-doped 1,3-bis(9-carbazolyl) benzene (mCP). It was quite stable and shifted slightly from (0.312, $0.398)$ at $1000 \mathrm{~cd} \mathrm{~m}^{-2}$ to $(0.320,0.395)$ at $9000 \mathrm{~cd} \mathrm{~m}^{-2}$. This indicated that the energy transfer process between the monomer and excimer molecules was highly prevented as there was no ground state absorption in the excimer molecules [33]. The inset made the normalized EL spectra of WOLED at different current densities. The excimer emission was almost unchanged with respect to the monomer emission with increasing current intensity.

\section{Standard RGB Configuration}

Given the problem of luminance efficiency (LE) and other challenges such as charge balance and efficiency roll off, CRI of excimer-based single molecular WOLED architectures remains below values required for solid-state lighting, i.e., below 80. Currently, intense research interest is still focused on the classic approach to fabricating WOLED by employing multi-emission layer structures in which two complementary colors or three primary colors (blue, green, and red) are emitted from different organic layers.

Recent results have shown some WOLEDs employing ultra-thin EMLs [34-36]. They have successfully reduced efficiency roll-off based on a carrier and exciton-confining structure, and their 
contribution to the efficiency and stability of WOLEDs. However, due to the sensitive nature of ultra-thin layers, a slight variation in them can bring about obvious effect on the performance of WOLED. Therefore, Yu et al. varied the thickness ratio between ultra-thin red and green EMLs, keeping total thickness of two EMLs at $1 \mathrm{~nm}$, to fabricate Devices A, B and C (shown in Table 1) [37].

Table 1 Layer setup, EL performance, and chromaticity for each investigated sample.

\begin{tabular}{ccccccc}
\hline Device & $\begin{array}{c}\text { Red EML } \\
\text { thickness }(\mathrm{nm})\end{array}$ & $\begin{array}{c}\text { Green EML } \\
\text { thickness }(\mathrm{nm})\end{array}$ & $\begin{array}{c}\text { Max.PE } \\
\left(\mathrm{lm} \mathrm{W}^{-1}\right)\end{array}$ & $\begin{array}{c}\text { Max.PE } \\
\left(\mathrm{cd} \mathrm{A}^{-1}\right)\end{array}$ & $\begin{array}{c}\mathrm{LE}\left(\mathrm{cd} \mathrm{A}^{-1}\right) \\
\text { @1000cd m }\end{array}$ & CRI \\
\hline A & 0.25 & 0.75 & 22.9 & 29.2 & 22.8 & 80 \\
B & 0.50 & 0.50 & 25.8 & 28.8 & 21.9 & 66 \\
C & 0.75 & 0.25 & 21.5 & 23.9 & 17.5 & $<40$ \\
\hline
\end{tabular}

The EL spectra measured at $1000 \mathrm{~cd} \mathrm{~m}^{-2}$ of Devices A, B and C are shown in Fig. 3. It has been found that Device B shows almost the same efficiency characteristic as Device A. The maximum LE of $28.8 \mathrm{~cd} \mathrm{A-1}$ and PE of $25.8 \mathrm{~lm} \mathrm{W-1}$, falling to $21.9 \mathrm{~cd} \mathrm{A-1}$ and $10.6 \mathrm{~lm} \mathrm{~W}-1$ at $1000 \mathrm{~cd} \mathrm{~m}^{-2}$, respectively. The higher green and blue emission intensities lead to a lower CRI of 66. The red EML thickness of Device $\mathrm{C}$ is the thickest among all OLEDs employing ultra-thin EMLs, which results in more excitons confined in red EML. Affected by the insufficient blue and green emission intensities, the very low CRI value in Device $C$ is obtained (see Table 1). Analysis has shown that it is very essential to fine-tune thicknesses of EMLs in a WOLED employing ultra-thin EMLs in order to realize high efficiency and CRI value.

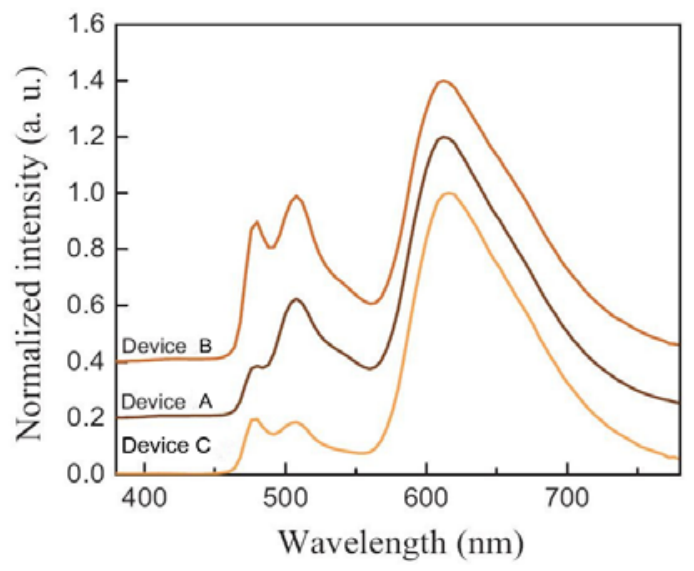

Fig. 3. EL spectra of Devices A, B and C at $1000 \mathrm{~cd} \mathrm{~m}^{-2}$.

It is also well known that, to realize high CRI, a WOLED should have an as broad as possible emission spectrum. The WOLED shown in Fig. 4 employs a long lifetime blue fluorophor as well as the high efficiency green/red phosphor [12]. The emission spectrum is primarily due to the red emitter $(\lambda$ peak $=612 \mathrm{~nm})$ and the green emitter $(\lambda$ peak $=512 \mathrm{~nm})$, with relatively weaker intensity of blue. The result indicates that the recombination zone is located at the interface between red and green emitting layers. Similarly, the recombination zone extends to red EML with increasing electric field, leading to the slight reduction of green and blue emission with the increasing brightness. Even so, this device still exhibits high CRI values above 85 at each luminance level (see Table 1). The mechanism responsible for these high CRI was the appropriate intensity match among the three primary colors. 


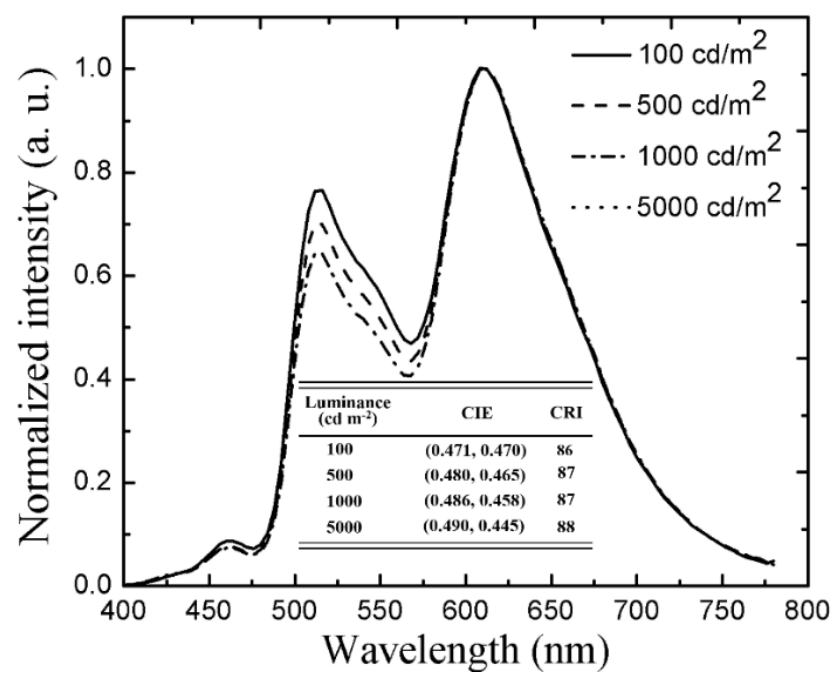

Fig. 4. The normalized EL spectra of the RGB WOLED at 100, 500, 1000, and $5000 \mathrm{~cd} \mathrm{~m}^{-2}$ respectively. The inset is the summary of chromatic performance characteristics for RGB WOLED.

\section{Multi-Peak Spectrum Emission}

To gain wide acceptance in the high-quality white-light illumination, an ideal WOLED should mimic the spectral distribution of natural sun light. To obtain these spectral characteristics, a range of different approaches has been investigated in the past. It is the most commonly used approach to obtain sunlight-style OLED by composing red, green, yellow and blue emission in a device.

While the example discussed above already employs multi-waveband WOLED based on multi-emission layers' structures to achieve a CRI approaching sunlight, the device discussed in the following combines emission (see Fig. 5) [12]. The spectrum of Sun-like device measured at $5000 \mathrm{~cd}$ $\mathrm{m}^{-2}$ appears to be more saturated than sunlight, even though it has only four wavebands. Moreover, the high-CRI of 89 was obtained for Sun-like device. The fabrication of WOLED focuses on the role of the yellow component as it plays a crucial role in enhancing the quality of the lighting source to mimic sunlight.

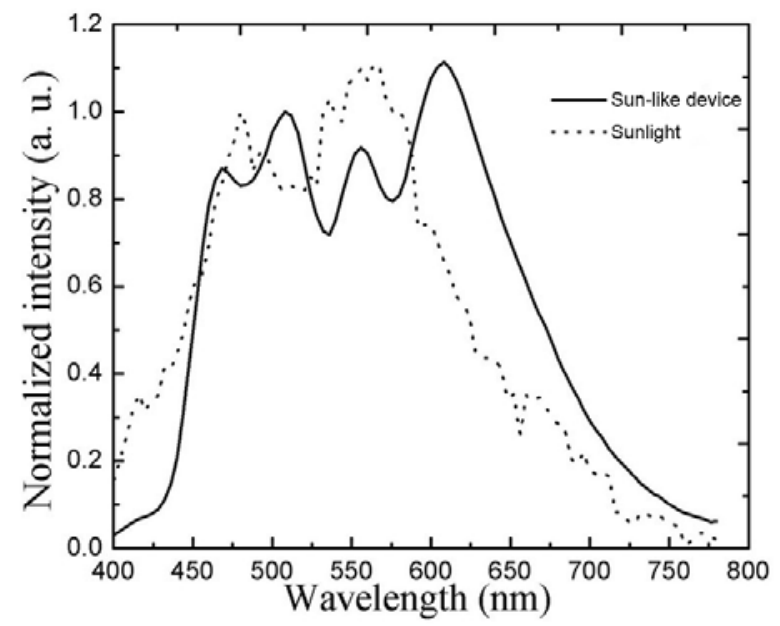

Fig. 5. The spectra comparison between Sun-like device and sunlight.

Despite this success, as more emitters are incorporated to obtain high-CRI WOLEDs, problems also appear in the form of more complex structure, potential higher cost and color shift. But the utilization of excimer and exciplex emission provides a particularly interesting approach to achieve white emission with high-CRI due to its decreased number of emissive dopants and simple device 
geometry [38]. For this reason, we will focus the following discussion on high-CRI WOLED by the incorporation of yellow or green fluorescence emission located between the monomer and excimer emission of Pt-4 [39].

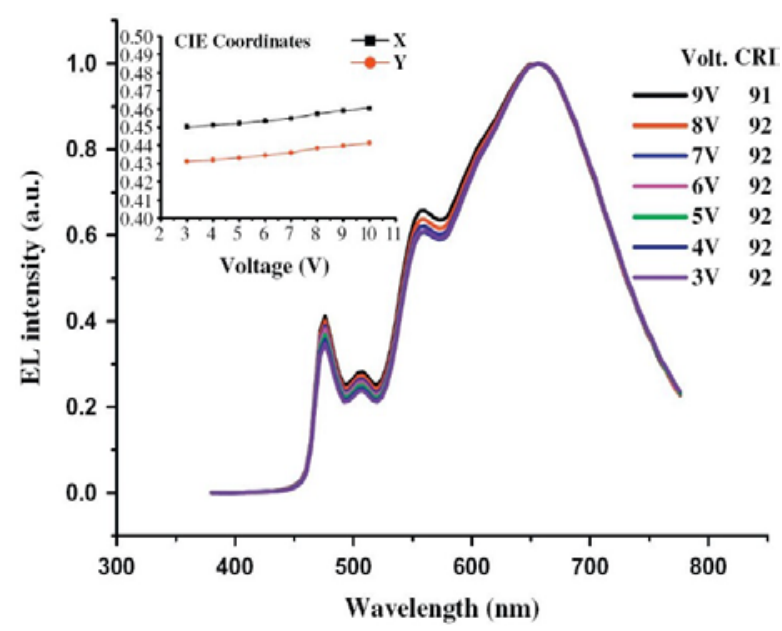

Fig. 6. The EL spectra and CRI at various driving voltages for the device with $0.5 \mathrm{~nm}$ interlayer. The inset show the CIE coordinates variation of the device.

Pt-4 is a molecule demonstrating light-blue monomer emission and red excimer emission, which has been proved to obtain WOLEDs with a single emissive layer (see Section 3). Figure 6 show changes of the emission spectrum and chromaticity with driving voltage. Besides the effect of relative emission intensity adjustment to obtain a high CRI, the mCP interlayer of the architecture also plays an important role in the chromatic stability of the device. It can be seen from Fig. 6 that for the device with $0.5-\mathrm{nm}$ interlayer, the variation of the spectrum is slight with the increasing driving voltage from $3 \mathrm{~V}$ to $9 \mathrm{~V}$, and the CIE coordinates changes from $(0.450,0.431)$ to $(0.459,0.439)$. The excellent chromatic stability is attributed to the suppression of the shift of excited recombination zone under the effect of the interlayer [40]. These results demonstrate that the four-peak WOLED has great potential for development as an OLED in virtue of its sunlight-like qualities and could meet the requirements for lighting in dwellings.

\section{Summary}

High quality WOLEDs have been rapidly increasing levels of attention in academia over the past years and their performance has greatly improved, particularly in terms of color stability and CRI. Owing to the fact that about $20 \%$ of the internally generated photons are trapped within the anode/organic-guided modes, a first focus is put on reviewing promising concepts for improved light outcoupling. A light-emitting layer (EML) of WOLED, consisting of 8 wt\% Pt-4-doped 1,3-bis(9-carbazolyl) benzene (mCP), was very stable and shifted slightly from $(0.312,0.398)$ at 1000 $\mathrm{cd} \mathrm{m}^{-2}$ to $(0.320,0.395)$ at $9000 \mathrm{~cd} \mathrm{~m}^{-2}$. Nevertheless, it is still unclear whether CC-HIL approach will ultimately reach the best balance of carrier, higher efficiency and long-term stability. While for a single emitting substance, excimer-based emission route is an alternative method, for fabricating high-efficiency, chromatically stable WOLEDs. However, the dominating approach is to combine the EL from a set of different lumiphores, typically three or four. Overall, it now becomes increasing clear that the characteristics of the final device are not fundamentally related to the fabrication method used.

Further development of light source, it has been found that it is challenging to maintainsunlight-style color and a good CRI over a wide range of luminance levels, and future work investigating this phenomenon should be accelerated. Although there are significant challenges over 
light source, we are optimistic that OLED technology will meet practical goals and expectations in the future.

\section{Acknowledgement}

This work was supported by the Special Appointment (Eastern Scholar) at Shanghai Institutions of Higher Learning, the National Natural Scientific Foundation of China (61136003, 61275041), the “973”program(2015CB655005), and the Project of National Post-Doctor Fund (2015M580315).

\section{References}

[1] C. W. Tang, S. A. VanSlyke. Appl. Phys. Lett. 51 (1987) 913-915.

[2] A. Köhler, J. S. Wilson. Adv. Eng. Mater. 4 (2002) 453-459.

[3] N. Thejokalyani, S. J. Dhoble. Renew. Sust. Energ. Rev. 32 (2014) 448-467.

[4] S. R. Forrest, Nature. 428 (2004) 911-918.

[5] O. Lavastre, L. Lllitchev. J. Am. Chem. Soc. 124 (2002) 5278-5279.

[6] J. Shi, C. W. Tang. Appl. Phys. Lett. 80 (2002) 3201-3203.

[7] H. Kanno, K. Ishikawa. Appl. Phys. Lett. 90 (20071) 123509-123509.

[8] B. W. D’Andrade, S. R. Forrest. Adv. Mater. 16 (2004) 1585-1595.

[9] Wei Liu, Rongming Wang, and Ning Wang, Appl. Phys. Lett. 97 (2010) 041916.

[10]Wei Liu, Ning Wang, and Rongming Wang, Nano Lett., 11 (2011) 2983-2988.

[11]D. Qin, Y. Tao. Appl. Phys. Lett. 86 (2005) 113507-113507.

[12]J. Yu, B. Wei. J. Mater. Chem. 22 (2012) 22097-22101.

[13]T.W. Lee, J. Kido. Appl. Phys. Lett. 92 (2008) 043301-043301.

[14]P. J. Alessi, E. C. Carter, Commission Internationale de L’éclairage (CIE). Colorimetry, Publication Report No. 15.2. (1986)

[15]W. Münch, M. Richter, Commission Internationale de L’éclairage (CIE), Publication Report No. 13.2 (1974).

[16]S. Reineke, M. Thomschke. Rev. Mod. Phys. 85 (2013) 1245.

[17]M. C. Gather, A. Köhnen. Adv. Mater. 23 (2011) 233-248.

[18]J. Kido, K. Hongawa. Appl. Phys. Lett. 64 (1994) 815-817.

[19]Q. Wang, J. Q. Ding. Appl. Phys. Lett. 94 (2009) 103503.

[20]E. L. Williams, K. Haavisto. Adv. Mater. 19 (2007) 197-202.

[21]J. H. Jou, Y. S. Chiu. Appl. Phys. Lett. 88 (2006) 193501.

[22]S. Tao, C. S. Lee. Appl. Phys. Lett. 91 (2007) 013507-013507.

[23]Y. C. Tsai, J. H. Jou. Appl. Phys. Lett. 89 (2006) 243521.

[24]Y. G. Lee, I. S. Kee. Appl. Phys. Lett. 90 (2007) 243508-243508.

[25]G. Gu, G. Parthasarathy. J. Appl. Phys. 86 (1999) 4067-4075.

[26]F. W. Guo, D. G. Ma. Appl. Phys. Lett. 87 (2005) 173510-173510.

[27]B. C. Krummacher, V. E. Choong. Appl. Phys. Lett. 88 (2006) 113506. 
[28] M. Pempointner. J. Phys. D: Appl. Phys. 43 (2010) 205102.

[29]H. Xu, B. Wei. J. Phys. D: Appl. Phys. 44 (2011) 415102.

[30]J. Brooks, Y. Babayan. Inorg. Chem. 41 (2002) 3055-3066.

[31]H.-F. Xiang, S.-C. Chan. Chem. Commun. 11 (2005) 1408-1410.

[32]L. Murphy, P. Brulatti. Chem. Commun. 48 (2012) 5817-5819.

[33]B. W. D’Andrade, J. Brooks. Adv. Mater. 14 (2002) 1032-1036.

[34]S. J. Su, E. Gonmori. Adv. Mater. 20 (2008) 4189-4194.

[35]L. Li, J. Yu. J. Lumin. 128 (2008) 1783-1786.

[36]J. Yu, W. Zhang. Displays. 32 (2011) 87-91.

[37]J. Yu, B. Wei. Phys. Status Solidi A. 210 (2013) 408-412.

[38]X. Yang, Z. Wang. Appl. Phys. Lett. 93 (2008) 193305.

[39]L. Xiong, W. Zhu. Org. Electron. 14 (2013) 32-37.

[40]S. Reineke, F. Lindner. Nature. 459 (2009) 234-238. 